\title{
Complex pattern formation in reaction-diffusion systems with spatially varying parameters
}

\author{
Karen M. Page ${ }^{\mathrm{a}, *}$, Philip K. Maini ${ }^{\mathrm{b}}$, Nicholas A.M. Monk ${ }^{\mathrm{c}, 1}$ \\ ${ }^{a}$ Bioinformatics Unit, Department of Computer Science, University College London, Gower Street, London WC1E 6BT, UK \\ b Centre for Mathematical Biology, Mathematical Institute, 24-29 St. Giles', Oxford OX1 3LB, UK \\ ${ }^{\mathrm{c}}$ Centre for Bioinformatics and Computational Biology, Division of Genomic Medicine, University of Sheffield, \\ Royal Hallamshire Hospital, Sheffield S10 2JF, UK
}

Received 23 September 2003; received in revised form 14 October 2004; accepted 28 January 2005

Available online 5 March 2005

Communicated by A. Doelman

\begin{abstract}
Spontaneous pattern formation in reaction-diffusion systems on a spatially homogeneous domain has been well studied. However, in embryonic development and elsewhere, pattern formation often takes place on a spatially heterogeneous background. We explore the effects of spatially varying parameters on pattern formation in one and two dimensions using the Gierer-Meinhardt reaction-diffusion model. We investigate the effect of the wavelength of a pre-pattern and demonstrate a novel form of moving pattern. We find that spatially heterogeneous parameters can both increase the range and complexity of possible patterns and enhance the robustness of pattern selection.
\end{abstract}

(C) 2005 Elsevier B.V. All rights reserved.

Keywords: Spatially varying parameters; Gierer-Meinhardt reaction-diffusion; Turing space; Pattern formation; Self-organised spatial pattern

\section{Introduction}

The behaviour of a wide range of nonlinear dynamical systems is characterised by the ability to form spontaneous stable spatial patterns from an initial unpat-

\footnotetext{
* Corresponding author. Tel.: +44 2076793683 ; fax: +44 2073871397 .

E-mail addresses: k.page@cs.ucl.ac.uk (K.M. Page), maini@maths.ox.ac.uk (P.K. Maini),n.monk@sheffield.ac.uk (N.A.M. Monk).

1 Present address: Department of Computer Science, University of Sheffield, Regent Court, Sheffield S1 4DP, UK.
}

terned state [8]. Significant progress has been made in understanding the mechanisms that underlie patterning, and in determining the dependence of the final pattern on model parameters, initial and boundary conditions, and domain size and growth (see for example [18,7]). One class of model that has been studied in detail is based on the Turing instability in reaction-diffusion systems [30]. Turing demonstrated that a system of two or more mutually interacting diffusible chemicals (termed morphogens) can undergo spontaneous spatial patterning within a specific region of parameter space (the Turing space). Such Turing 
patterns have since been produced experimentally in chemical systems (reviewed, for example, in $[9,18]$ and articles in [16]).

Typically, the analysis of pattern-forming models is carried out assuming that both the initial conditions and model parameters are homogeneous in space. In the case of reaction-diffusion models, this yields, in principle, a complete characterisation of the Turing space (see, for example [21,22]). In reality, however, pattern-forming mechanisms often operate in spatially heterogeneous environments. In such cases it becomes critical to understand the effects of the imposed spatial heterogeneity on the ability of the mechanism to generate pattern, and on the form of the resulting pattern.

One specific instance in which spatial heterogeneity is significant is in the emergence of pattern during embryonic development, or morphogenesis [13]. Specifically, development proceeds in a hierarchical fashion, with pattern being laid down in progressively finer detail as the embryo ages and grows. At each stage of this process, the biological patterning mechanisms operate within a cellular environment that already bears the imprint of earlier patterning events. This may be manifested in differences both in the internal state of cells, and in the environment external to the cells (for example, the extracellular matrix). Given that cellular and extracellular conditions can modulate the parameters and initial conditions of patterning mechanisms, it seems clear that the possibility of spatially inhomogeneous parameters should be considered in models of such mechanisms.

In this paper, we investigate the effect of spatially varying parameters in a two-component reactiondiffusion system. We study the well-characterised activator-inhibitor model introduced by Gierer and Meinhardt [12]. In a previous paper [26], we demonstrated that spatially varying parameters in this model can lead to patterning outside of the Turing space and that the patterns generated can be spatially restricted. We now explore further the patterning capabilities of this model system with spatially varying parameters. In particular, we determine how the variation of different parameters in the model equations affects the steady state patterns formed. Using the insight gained, we determine the effects on pattern formation of varying the spatial wavelengths of "pre-patterns" in the model parameters. We show that spatial variation in model parameters can result either in complex stable patterns, or in novel propagating patterns. Finally, we extend our study to a two-dimensional model to illustrate the wealth and complexity of patterns that can be generated with a spatially varying parameter and discuss possible applications in biology. Patterning in two dimensions is of great significance during embryonic development, since many pattern-forming events (such as the specification of neurons in the fly neuroectoderm [6]) take place in epithelial sheets which have an (approximately) two-dimensional structure.

\section{The dependence of spatial patterns on model parameters}

As a first step towards understanding the effects of spatial inhomogeneity in model parameters, we need to assess how each model parameter affects the form of the final steady state pattern. For concreteness, we focus on the two-component activator-inhibitor system of reaction-diffusion equations introduced by Gierer and Meinhardt [12]. In a non-dimensional form [26], the model equations are

$u_{t}=r\left(1+\frac{u^{2}}{v}\right)-\mu u+D_{u} \nabla^{2} u$,

$v_{t}=r u^{2}-v v+D_{v} \nabla^{2} v$,

where $u(\mathbf{x}, t)$ and $v(\mathbf{x}, t)$ denote, respectively, the activator and inhibitor concentrations at spatial position $\mathbf{x}$ and time $t . r, \mu, v, D_{u}$ and $D_{v}$ are positive parameters. These equations are solved on a specified spatial domain with given boundary conditions (we assume zero flux). When the model parameters are within the Turing space, stable spatially patterned steady state solutions exist for $u$ and $v$, in which localised peaks of activator concentration are separated by a characteristic spacing [12].

In general, it is difficult to determine the effect on patterning of each model parameter analytically. From standard linear theory we know that the activator diffusion coefficient $D_{u}$ must be smaller than the inhibitor diffusion coefficient $D_{v}$ for diffusion-driven instability, so we assume that $D_{u}$ is very much smaller than the $D_{v}$ and set $D_{u}=\epsilon^{2} \times D_{v}$, where $\epsilon$ is a small parameter, and we seek a solution of (1) for $u$ and $v$ in terms of a series expansion in $\epsilon$. However it is not possible to construct a regular series expansion which satisfies the dif- 
ferential equations and both boundary conditions. We thus consider an "outer expansion" which is regular and satisfies the equations in most of the domain together with "inner expansions" which match with the outer solution and satisfy the equations in small boundary layer regions of the domain. In these regions the coefficients of the series solution are given in terms of a rescaled spatial coordinate. This matched asymptotic analysis, which is described for a one-dimensional system in Appendix A, shows that when $d \equiv D_{u} / D_{v}$ is $\mathrm{O}\left(\epsilon^{2}\right)$, the
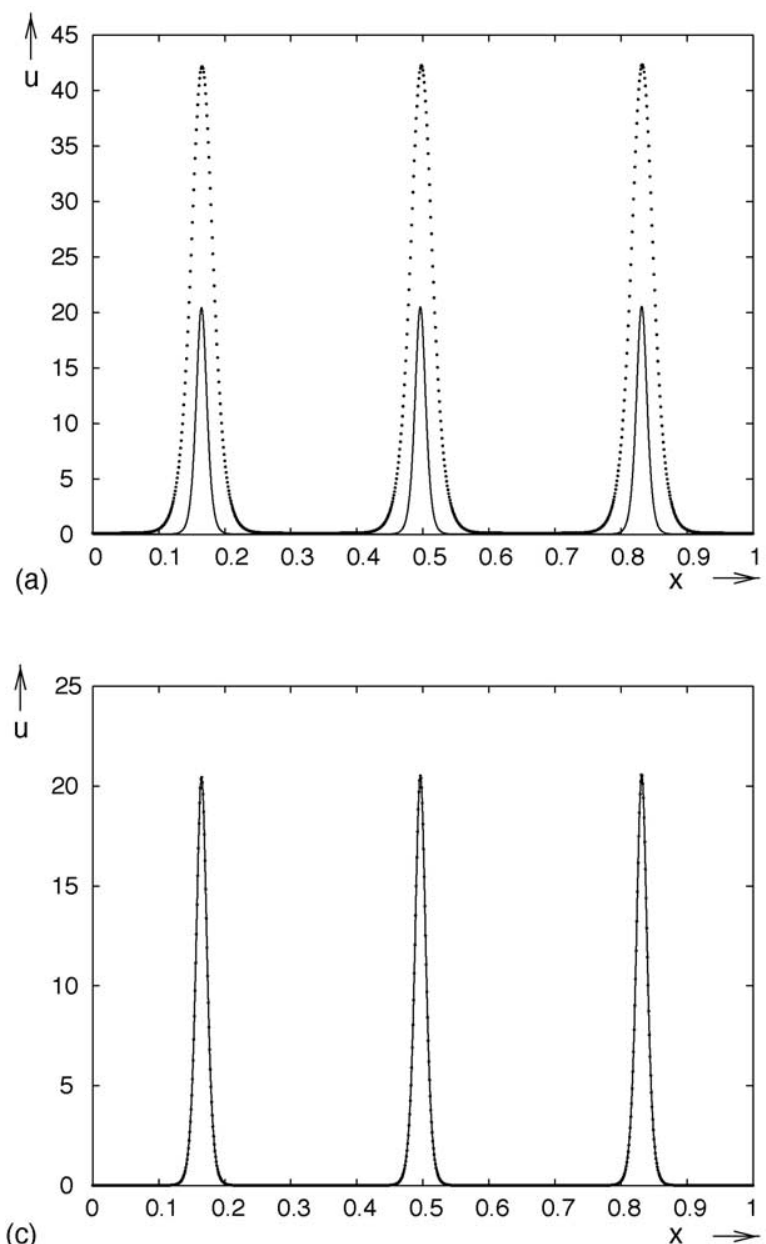

width of the activator peaks is proportional to the diffusion wavelength of the activator $\lambda_{u} \equiv \sqrt{D_{u} / \mu}$. The length-scale of the inhibitor pattern (which gives us an idea of the spacing of the activator peaks) is of order the diffusion wavelength of the inhibitor $\lambda_{v} \equiv \sqrt{D_{v} / v}$. The heights of the activator peaks are proportional to $\xi=\sqrt{D_{v} v / D_{u} \mu}$.

Fig. 1 shows the results of simulations of the GiererMeinhardt model equations in one spatial dimension. Fig. 1(a) shows the steady state activator profiles in two
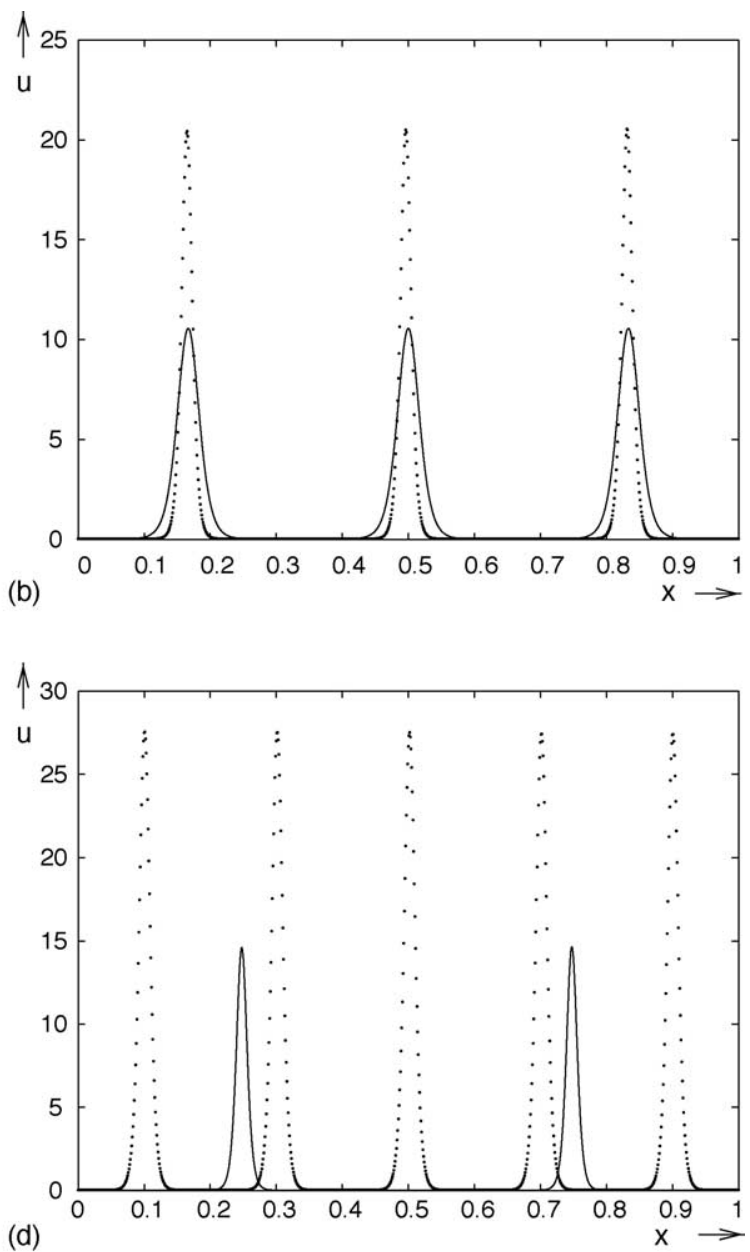

Fig. 1. Steady state activator profiles in simulations of the Gierer-Meinhardt model equations. In all simulations $D_{v}=0.027$. In (a) $d=$ $0.001, r=0.03$ and $v=2.0$. The dotted line corresponds to the solution when $\mu=0.25$ and the solid line corresponds to the solution when $\mu=1.0$. In (b) $r=0.03, v=2.0, \mu=1.0$ and $d=0.001$ (dotted line) $/ d=0.004$ (solid line). In (c) $v=2.0, \mu=1.0, d=0.001$ and $r=0.04$ (dotted line) $/ r=0.01$ (solid line). In (d) $r=0.03, \mu=1.0, d=0.001$ and $v=4.0$ (dotted line) $/ v=1.0$ (solid line) [numerical solver-NAG library routine: $\mathrm{D} 03 \mathrm{PCF}$, number of discretization points $=1001$, tolerance $=10 \mathrm{E}-07$, initial conditions $\pm 10 \%$ noise around the uniform steady state in each case]. 
simulations with different values of $\mu$, the other parameters being held constant (parameter values given in legend). As expected, quadrupling the value of $\mu$ roughly halves the height and width of the peaks and does not change their separation. In Fig. 1(b), two different values of $d$ or equivalently $D_{u}$ were used. Quadrupling $D_{u}$ roughly doubles the width and halves the height of the peaks. In Fig. 1(c) two values of $r$ were used. The steady state activator profiles are almost identical (but the height of the inhibitor profile changes roughly in direct proportion to $r$ ). In Fig. 1(d) two values of $v$ were used. We see that quadrupling $v$ approximately doubles the height and halves the separation of the activator peaks, but has no discernible effect on their width. These simulation results all agree with the analytical expressions we have found for the widths, heights and separations of the peaks.

In summary, the separation of the peaks is unaffected by the values of $\mu, r$ and $d$ and is proportional to $\lambda_{v}$. The width of the activator peaks is independent of the values of $v, r$ and $D_{v}$ and proportional to $\lambda_{u}$ and the height of the peaks is independent of $r$ and proportional to $\xi=\sqrt{D_{v} v / D_{u} \mu}$.

The matched asymptotic analysis suggests that a complex pattern of peaks of varying heights, widths or separations could be produced in a reaction-diffusion system in which one of the parameters was controlled by another chemical species which was itself patterned, for example by a different reaction-diffusion mechanism. The form of the resulting complex pattern would depend on which parameter was modulated by the control species.

\section{Spatially varying parameters in one dimension}

\subsection{Monotonically varying parameters}

Now that we have some insight into the effects that each of the parameters has on the final steady state pattern, we look at the patterns produced when these parameters are varied across the domain. We consider a one-dimensional form of the model system (1) and allow a single parameter to vary across the domain $[0$, 1] in such a way that the parameters are everywhere within the Turing space. We use base parameter values $D_{v}=0.027, d=0.01, v=20.0, \mu=10.0, r=$
5.0. We modulate each of these parameters, one at a time, by a factor $(1.0+0.5 \times \cos \pi x)$, so that the parameter decreases monotonically across the domain.

Fig. 2 shows the effect of variation of each parameter. In Fig. 2(a), $d$ is varied and, as expected, the steady state pattern has activator peaks which are wider and lower at high $d$. There is no detectable change in the spacing of the peaks. In Fig. 2(b), $v$ is varied and, again as expected, the spacing of the peaks decreases and their height increases as $v$ is increased, while the width of the peaks is approximately independent of $v$. When either $\mu$ or $r$ is varied, as in Fig. 2(c) and (d), respectively, a steady state pattern is not formed but rather a travelling wave of peaks is obtained. New peaks emerge at large $\mu$ (or small $r$ ) and disappear at small $\mu$ (or large $r$ ). The new peaks are created just inside the boundary (see for example the nascent peak on the right of Fig. 2(d)) and are annihilated just inside the opposite boundary. The patterns at $t=1000$ are shown in the figures. The variation in forms of the peaks is not quite as expected for a steady state pattern. The widths are approximately independent of $r$, and the width and heights of the peaks decrease with $\mu$, as expected. However, in addition, the heights of the peaks decrease with $r$ and the peak separations vary with both $\mu$ and $r$. These features are not suggested by the perturbation analysis for the steady state solutions. A full understanding of these phenomena would require more detailed analysis.

Intuitively, the reason for the travelling peaks in the systems with gradients in $\mu$ or $r$ seems to be that the level of the activator in the troughs of the pattern, which is given to leading order in our perturbation expansions by $r / \mu$, varies across the domain. This leads to diffusion of the activator across the trough from the side on which it is higher to the side on which it is lower (i.e. down a gradient in $r$, or up a gradient in $\mu$ ). Thus peaks would be receiving activator on their upper sides and losing activator on their lower sides and hence move up the gradient in trough activator level. Clearly, the same effect would not occur for variations in $v$ and in the diffusion coefficients. We are not aware that this method of obtaining moving peaks has been demonstrated in the literature. Kramer et al. [17], do postulate that a dynamic pattern would result in a system in which a parameter varied from within the Turing space at the centre of the domain to outside the Turing space in a different manner at each end. In addition, Voroney et al. [31], show the occurrence of travelling waves in a 

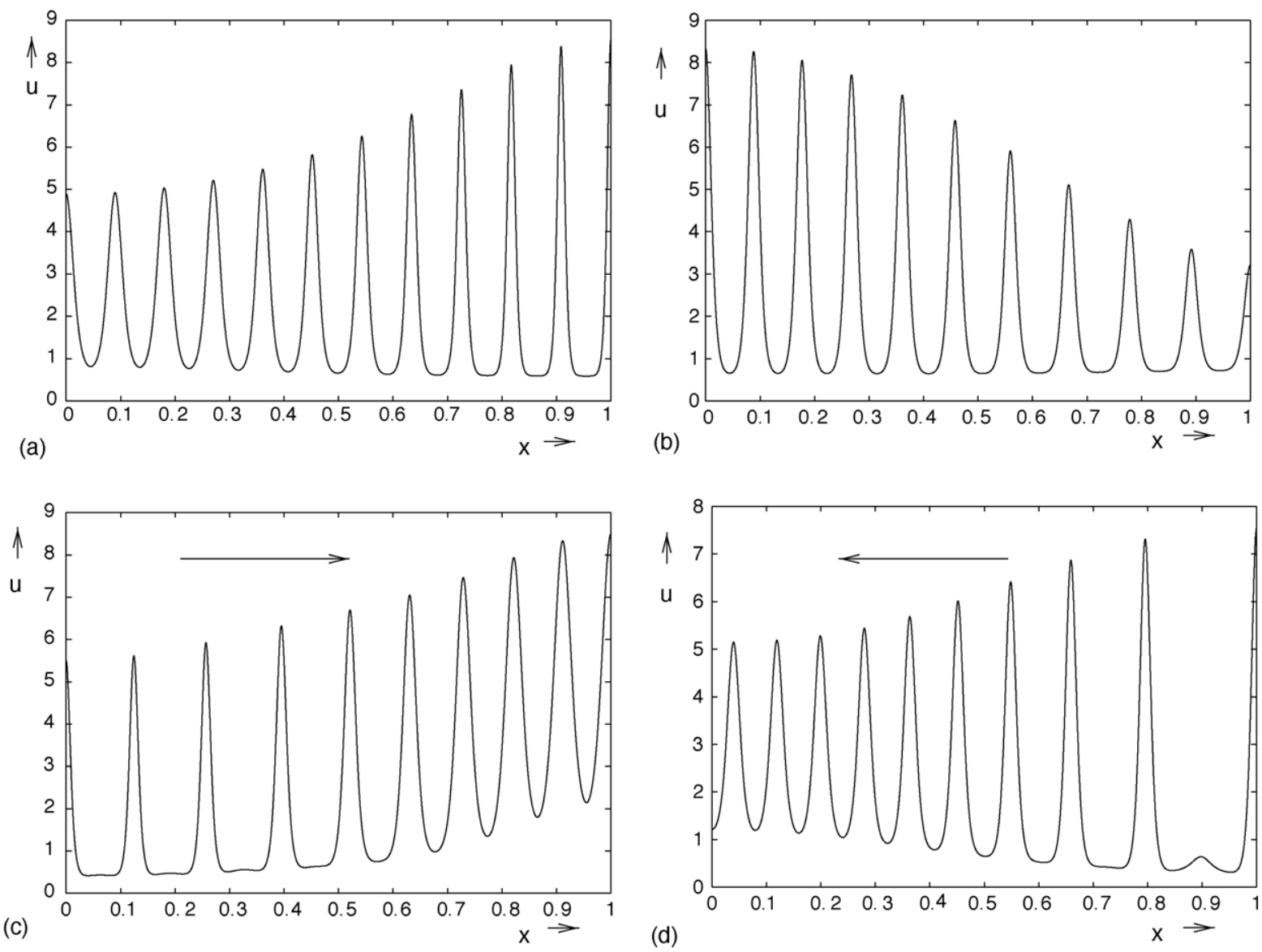

Fig. 2. Late time activator profiles in simulations of the Gierer-Meinhardt model equations in which a parameter varies across the domain. The values of the base parameters are $D_{v}=0.027, v=20.0, \mu=10.0, r=5.0$ and $d=0.01$. These base parameter values give rise to a pattern with many peaks, so that we can easily see how varying a parameter across the domain modulates the pattern. The mode with the largest linear growth rate is the $18 \pi$ mode. The peaks are also fairly smooth, which makes accurate numerical simulation easier: (a) $d=0.01(1.0+0.5 \cos \pi x)$; (b) $v=20.0(1.0+0.5 \cos \pi x)$; (c) $\mu=10.0(1.0+0.5 \cos \pi x)$; (d) $r=5.0(1.0+0.5 \cos \pi x)$. (a) and (b) show steady state activator profiles, whilst in (c) and (d) a snapshot of the moving profiles is shown [numerical solver-NAG library routine: D03PCF, number of discretization points $=1001$, tolerance $=10 \mathrm{E}-07$, initial conditions $\pm 10 \%$ noise around the uniform steady state of the system with the background parameters in each case].

reaction-diffusion system with a spatially varying parameter, but here again the parameter varies between a value within the Turing space and a value outside the Turing space, crossing a Turing-Hopf bifurcation. In contrast, in our simulations the parameters are within the Turing space at all points in the domain and hence if the parameter was constant at any of these values, the system would give rise to a stable spatial pattern.

To explore these moving peaks further, we consider matched asymptotic solutions for the full Gierer-Meinhardt model with the time derivative (see
Appendix B). We find that we can transform the boundary layer equation for $u$ (which to leading order is $\overline{u_{0}} / \epsilon$ ) to obtain Fisher's equation (see Appendix B):

$w_{t^{\prime}}=w(1-w)+w_{X^{\prime} X^{\prime}}$

where $\quad t^{\prime}=\mu t, X^{\prime}=\sqrt{\mu / D_{v}} X \quad$ and $\quad w=$ $1-3 \overline{u_{0}} / 2 u_{\text {peak }}=1-r \overline{u_{0}} / \mu \epsilon v_{*}$. Thus we might expect travelling waves within the boundary layer in $w=1-3 \overline{u_{0}} / 2 u_{\text {peak }}$ even in the system with constant parameters (where $u_{\text {peak }}$ is the value of $\overline{u_{0}}$ at the peaks 
of the steady state pattern). It appears, however, that the pattern is pinned in place because the peaks are confined to the boundary layer (and must not disappear entirely) and the positions of the boundary layers are fixed by the symmetry of the equations. If we consider the system with, for example, $\mu=\mu(x)$, then the transformation $w=1-r \bar{u}_{0} / \mu \epsilon v_{*}$ yields extra terms due to the spatial derivatives of $\mu$. These, however, are not present in the leading order equations, since the variation in $\mu$ takes place on a spatial scale of $\mathrm{O}(1)$ and thus is negligible across the boundary layer. Thus the differential equations for the leading order boundary expansions are the same as for the case with constant parameters. The lack of symmetry (in the matching conditions, albeit not to leading order) seems to allow the boundary layer to move. Intuitively it may be explained thus: the "pressure" for the lower $u$-state to invade is greater on the side of the peak on which the outer solution in $u$ takes smaller value and hence (in order to preserve the required thickness of the boundary layer) the peaks move down the gradient in $\mu$ (the outer solution value of $u$ is $r / \mu$ to leading order). This analysis is merely suggestive; to understand fully the phenomenon of moving peaks and the significance of Fisher's equation for $u$ in the boundary layer would require further analysis.

\subsection{Spatially periodic parameters}

The above simulations demonstrate that two-species reaction-diffusion systems with spatially varying parameters can lead to complex steady state patterns and travelling waves of peaks. The stable patterns consist of peaks of variable width, height and separation, unlike the straightforward periodic patterns that are familiar in reaction-diffusion systems with constant parameters. ${ }^{1}$ We suggest that the spatial variation in a parameter could be due to a previous patterning process, such as a reaction-diffusion system in another chemical species, which then controls the decay, production or diffusion rate of one of the species in our system (as in [1]). To give a specific example of the type of process

\footnotetext{
${ }^{1}$ Although, in most cases, simulations of reaction-diffusion systems with constant parameters result in patterns of identical peaks, we note the interesting work of Doelman et al. [10] in which stable patterns of spikes alternating between two different heights were obtained in a simulation of an alternative version of the GiererMeinhardt model.
}

that could be described well by this type of formalism, we consider pattern formation during embryonic development. The patterning of the embryo is naturally hierarchical and most patterning events are likely to take place in a heterogeneous environment. As we have shown, heterogeneity in parameters of a reactiondiffusion system extends the range and type of patterns that can be produced. There is good experimental evidence that the mobility of chemical species involved in embryonic patterning can be modulated by the concentration of others. For example, retinoic acid, which is graded along the anterior-posterior axis of the developing chick limb bud, modulates the permeability of gap junctions between cells and hence the effective diffusion coefficients of morphogens which move through the gap junctions $[2,5]$. There is also evidence that elements of the extracellular matrix (ECM) modulate the diffusion of growth factors such as activin [29], which are prime candidates for embryonic morphogens [25]. Thus spatial inhomogeneity in the structure of the ECM could lead to spatially varying diffusion coefficients. Other studies have shown how the stability of morphogens is modulated spatially by specific receptors expressed on the surface of cells (see, for example [3]).

To explore the influence of prior patterning events, we investigate now the effect of the wavelength of a periodic pre-pattern in the activator diffusion coefficient in the Gierer-Meinhardt model system. We use parameter values $v=20.0, \mu=10.0, r=10.0, D_{v}=0.027$ and $d=0.01(1.0+0.5 \cos n \pi x)$, where $n$ is an integer, which we vary. When $n=0$ (i.e. when the parameters of the system are spatially homogeneous) the pattern formed has approximately $23 / 2$ peaks, equally spaced and of equal heights (the exact pattern depends on the initial conditions). We look at the effect on the pattern when $n \ll 23$, when $n$ is a few times smaller than 23 and when $n>23$. We use this "base" pattern because it has many peaks; similar results hold for other base patterns. Fig. 3 shows typical steady state activator profiles. Fig. 4 shows the Fourier components of the steady state profiles given in Fig. 3.

We find that when the wavelength of the parameter variation is much larger than the natural wavelength of the reaction-diffusion system, the pattern formed is roughly of the natural wavelength, but the amplitudes of the peaks are modulated by an envelope with the same wavelength as that of the parameter variation. In 

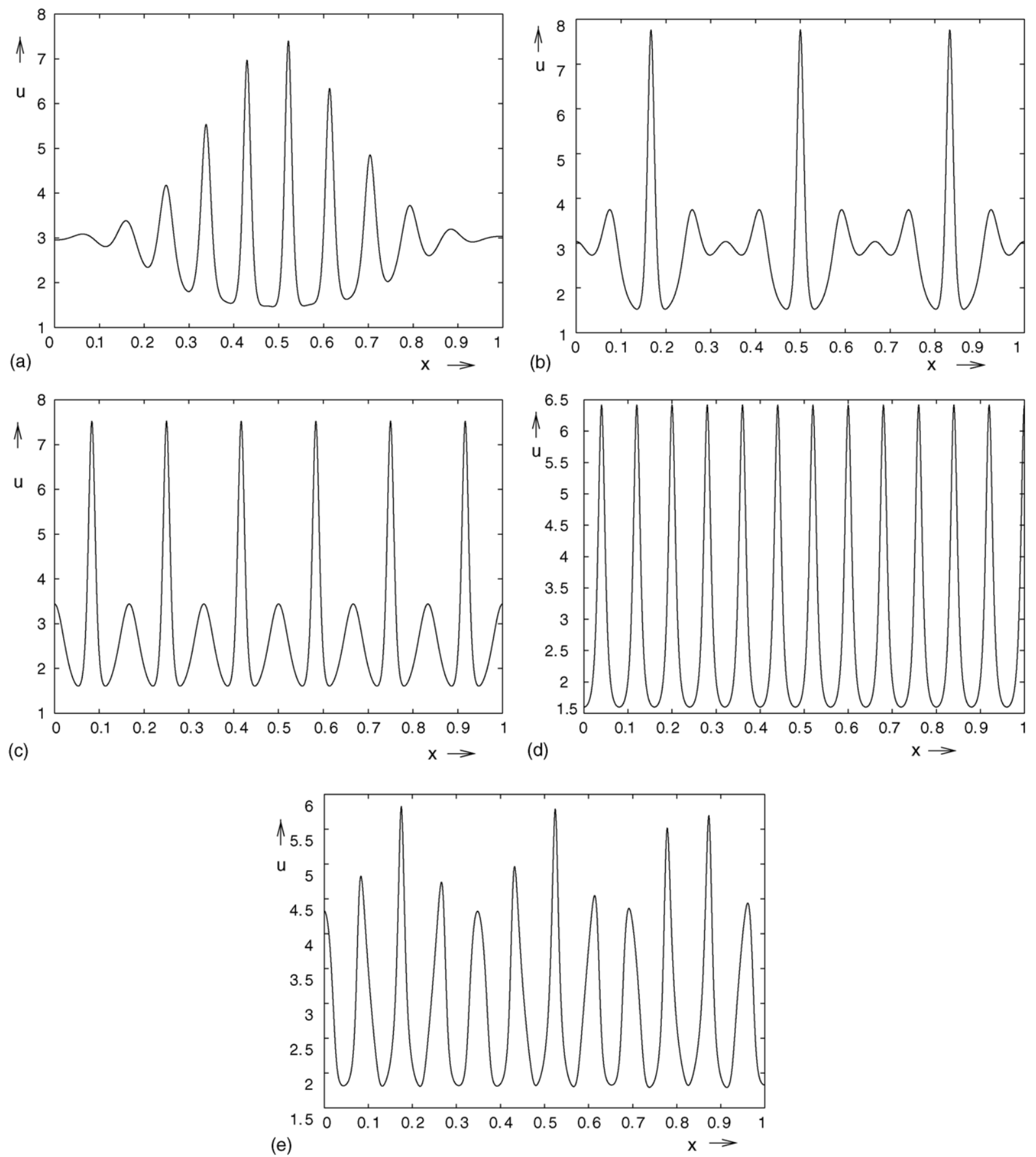

Fig. 3. Plot of the final steady state activator profiles in simulations of the Gierer-Meinhardt model equations with parameters $D_{v}=$ 0.027, $v=20.0, \mu=10.0, r=10.0$ and with $d$ varying sinusoidally in space. The wavelength of the pre-pattern in $d$ is different in each field. (a) $d=0.01(1.0+0.5 \cos 2 \pi x)$; (b) $d=0.01(1.0+0.5 \cos 6 \pi x)$; (c) $d=0.01(1.0+0.5 \cos 12 \pi x)$; (d) $d=0.01(1.0+0.5 \cos 25 \pi x)$; (e) $d=0.01(1.0+0.5 \cos 40 \pi x)$ [numerical solver-NAG library routine: D03PCF, number of discretization points $=1001$, tolerance $=10 \mathrm{E}-07$, initial conditions $\pm 10 \%$ noise around the uniform steady state of the system with the background parameters in each case]. 

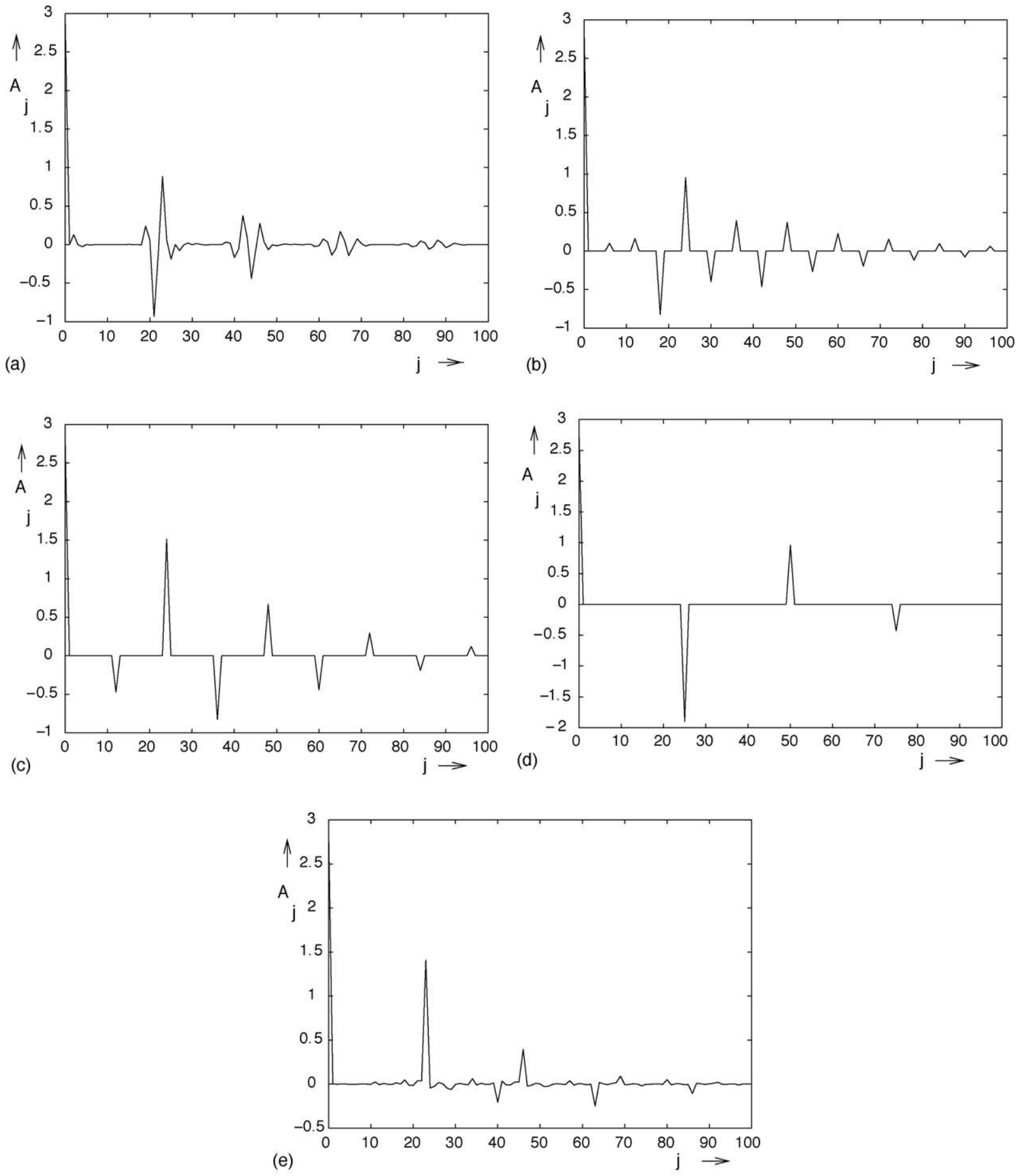

Fig. 4. Plots of the Fourier components $\left(A_{j}\right)$ of the solutions shown in Fig. 3 against their mode-numbers. 
the solution in Fig. 3(a), mode 23 and its harmonics are present (as in the case with constant parameters), but also mode $23 \pm 2$ and so on, which come from the interaction of the natural modes of the system and the parameter variation.

When the frequency of the parameter variation is larger, the only modes which appear in the solution are harmonics of the mode of the parameter variation. In Fig. 3(b), the only modes present are the harmonics of the $6 \pi$ mode. The mode with largest amplitude is the $24 \pi$ mode. This mode is closest to the natural wavelength of the reaction-diffusion system with constant parameters. The Fourier modes alternate in sign and give rise to a complex periodic pattern, whereas for the constant parameter case, a simple periodic pattern of identical peaks is produced, consisting of positive amplitudes of all the harmonics of mode 23. A similar Fourier mode behaviour is seen in Fig. 3(c) in which a pattern of alternating high and narrow, and low and wide peaks is formed, and in Fig. 3(d) where a pattern of identical peaks results. Fig. 3(e), like (a) has a more complex Fourier mode structure, in which mode 40 interacts with the harmonics of mode 23 to give rise to modes such as mode 63 . This again seems to give rise to a mode 23 pattern modulated by an envelope.
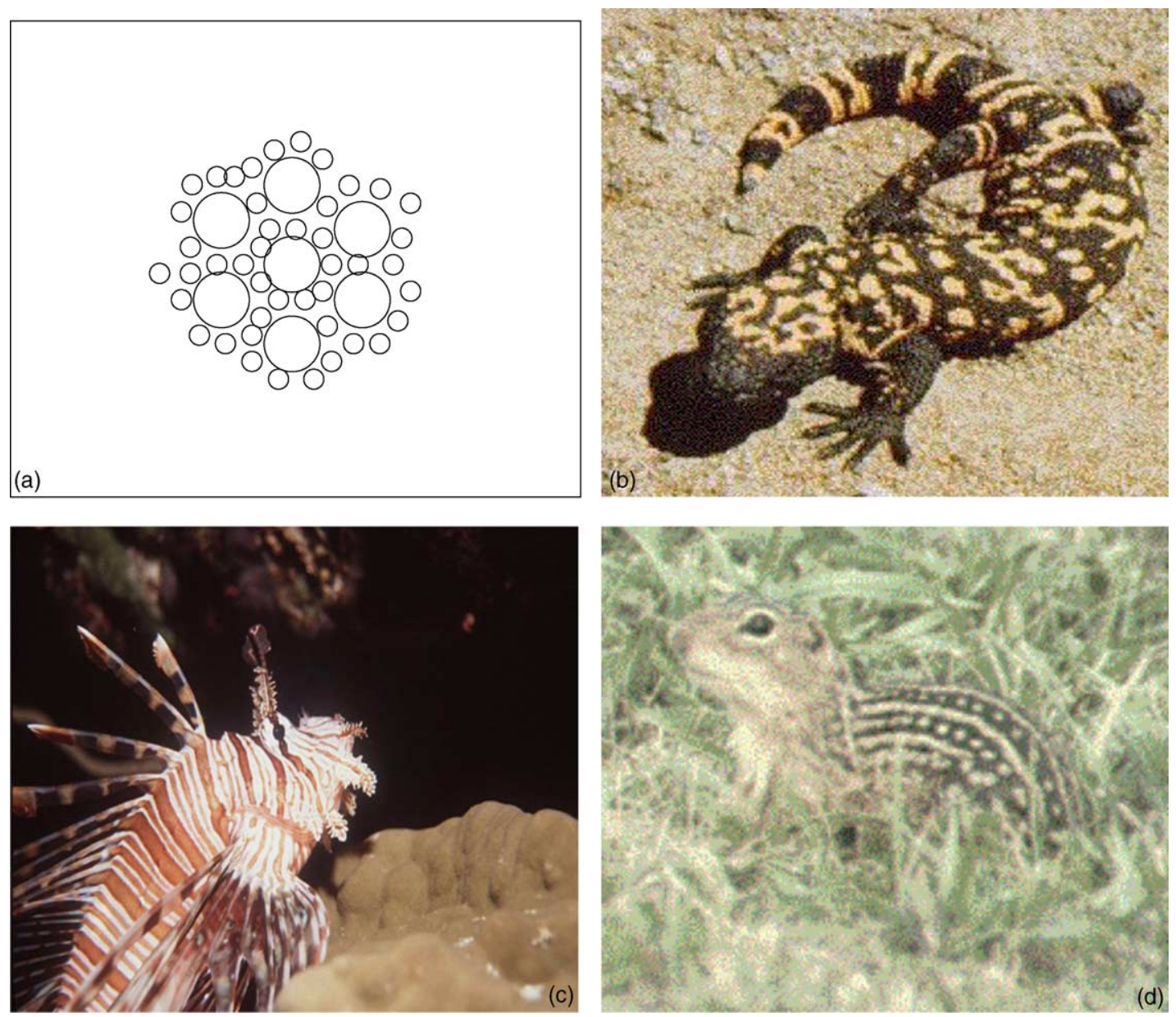

Fig. 5. Some examples of animals with interesting skin patterns. (a) The head of the lizard C. fedtschenkoi has a pattern of large and small scales (diagramatic representation); (b) the tail of the Gila monster has a pattern of alternating thick and thin dark stripes; (c) the skin of the Lionfish also shows alternating thick and thin dark stripes; (d) the 13-lined ground squirrel has a mixed pattern of spots and stripes. 

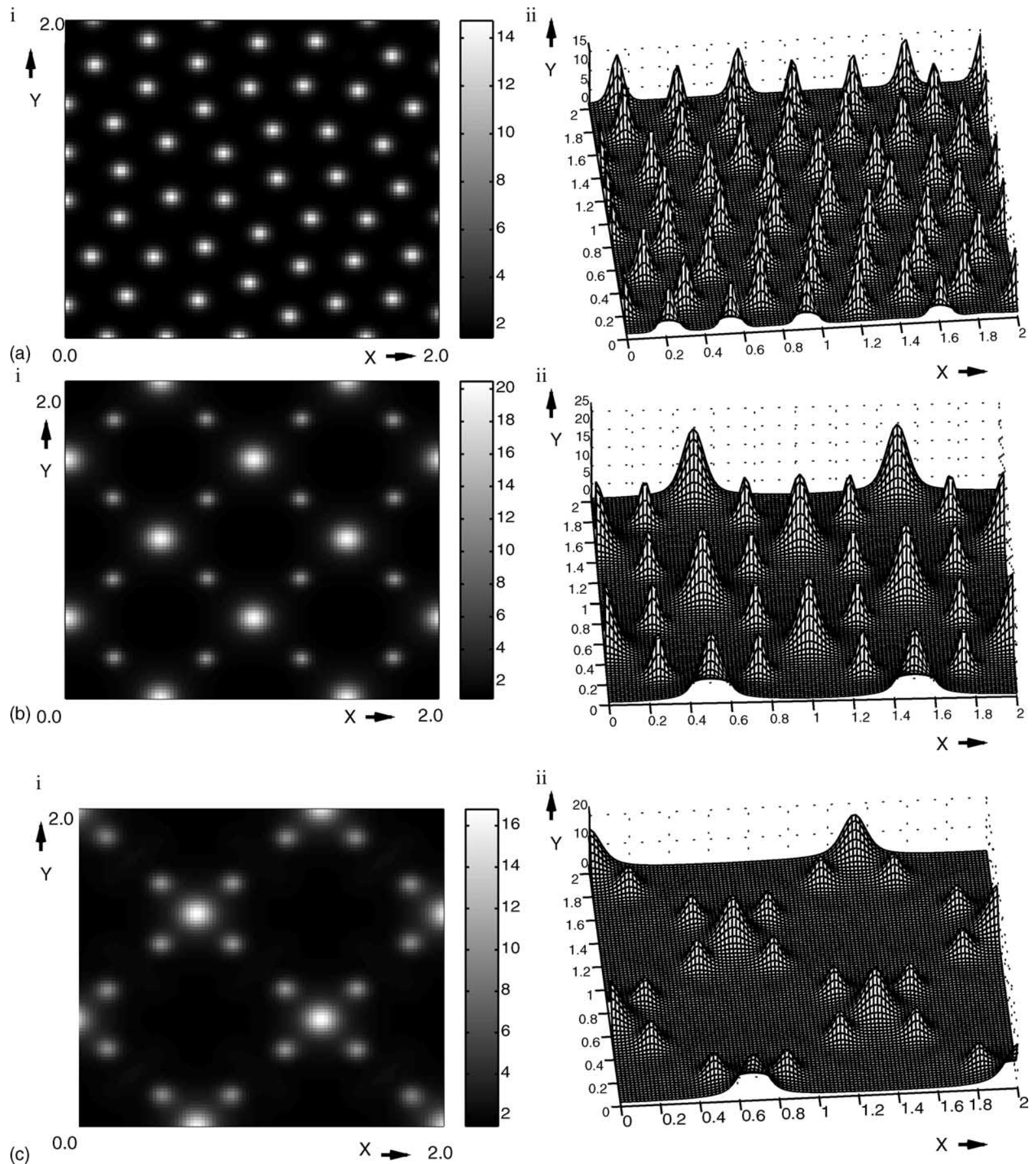

Fig. 6. Plot of the final steady state activator profiles in simulations of the Gierer-Meinhardt model equations on the domain [0, 2] $\times[0,2]$, with parameters $D_{v}=0.27, v=20.0, r=10.0, d=0.01$ and $\mu$ constant in (a) and varying sinusoidally in space in (b) and (c). (a) $\mu=10.0$; (b) $\mu=10.0(1.0+0.5 \cos 2 \pi x \cos 2 \pi y) ;($ c) $\mu=10.0(1.0+0.5 \cos 3 / 2 \pi x \cos 3 / 2 \pi y)$. Part (i) shows a greyscale plot of the solution and part (ii) a mesh plot [numerical solver-alternating direction implicit (ADI) method, timestep $=0.01$, spatial discretization step $=0.02$ ]. 

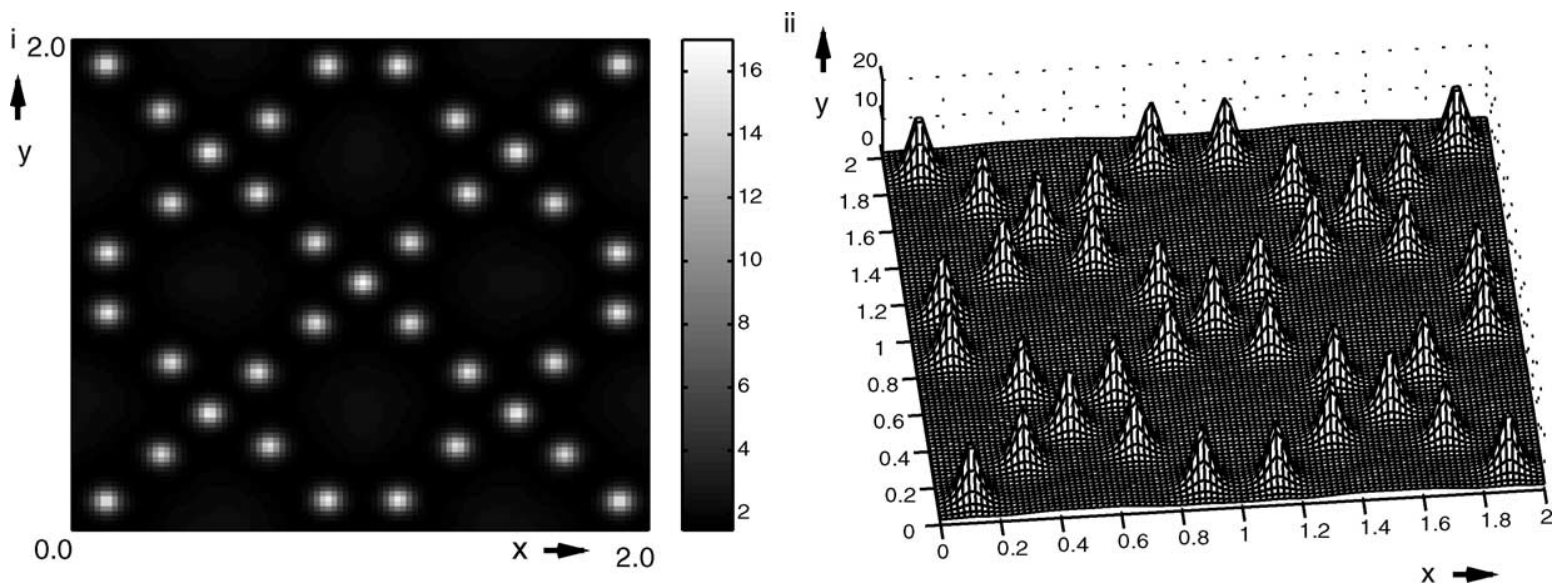

Fig. 7. Plot of the final steady state activator profiles in simulations of the Gierer-Meinhardt model equations on the domain $[0,2] \times[0,2]$, with parameters $D_{v}=0.27, \mu=10.0, r=10.0, d=0.01$ and $v=20.0(1.0+0.5 \cos 2 \pi x \cos 2 \pi y)$. Part (i) shows a greyscale plot of the solution and part (ii) a mesh plot [numerical solver-ADI method, timestep $=0.01$, spatial discretization step $=0.02$ ].

\section{Spatially varying parameters in two dimensions}

It is clear from the simulations in the previous section that a spatially varying parameter can enhance the complexity of patterns formed in a reaction-diffusion system. We now explore the types of patterns that may be produced with spatially varying parameters on a two-dimensional domain. Such systems could be involved in the patterning of embryonic epithelia, for example in the patterning of animal coats and fish skins, which are often characterised by complex spatial patterns. In particular, the profile of Fig. 3(c) suggests that it may be possible to obtain patterns of alternating large and small spots resembling the scale patterns on the head of the lizard Cyrtodactylus fedtschenkoi (see Fig. $5(a))$ or alternating wide and narrow stripes resembling those on the tail of the Gila monster (see Fig. 5(b)).

If we assume that cells differentiate where the activator concentration exceeds a certain threshold then the size of the differentiated region is controlled by the width of the activator peak. We have seen that this width is controlled by the diffusion and decay rates of the activator. Increasing the diffusion rate reduces the height of the peaks as it increases their width, implying that only for low thresholds of cell response will the wide, short peaks lead to larger patches of cell differentiation. Decreasing the parameter $\mu$ (the decay rate of the activator) increases both the height and the width of the peaks, so that any threshold of response below the height of the smaller peaks should lead to a mixed pattern of large and small patches of differentiation of significantly different sizes. Thus, in an attempt to obtain a pattern of differently sized peaks we vary $\mu$ across the domain. Fig. 6(a) shows a simulation of the Gierer-Meinhardt model equations (1) with constant parameters on the two-dimensional domain $[0,2] \times[0,2] .^{2}$ The parameter values are given in the legend. In Fig. 6(b) and (c), the details of the simulation are the same except that $\mu$ is varied sinusoidally in space (see legend for details). For each simulation we show (i) a greyscale plot which shows what the differentiated cell pattern might look like and (ii) a mesh plot which shows the form of the peaks. We see indeed that the largest peaks form at the minima of $\mu$ and that smaller peaks occur between these at medium values of $\mu$.

We find that it is possible to obtain a regular pattern of large and small spots (cf. Fig. 5(a)) and we postulate that, in a reaction-diffusion system that normally gives rise to a pattern of stripes (e.g. one with cubic autocatalysis) a spatially varying parameter could give rise to a pattern of thick and thin stripes, like the pattern on the skin of the lionfish (see Fig. 5c)).

\footnotetext{
${ }^{2}$ All two-dimensional simulations were performed using an alternating direction implicit (ADI) method [28]. Central finite differences were used for the spatial discretization.
} 
The pattern shown in Fig. 6(c) shows clusters of peaks, consisting of a larger peak surrounded by several smaller ones. This is similar to the pattern of hair follicles formed in many mammalian species. Nagorcka $[23,24]$ discusses the initiation of wool follicles in sheep and proposes a complex model, in which the formation of the first follicles modulates the domain and diffusion coefficients of a reaction-diffusion system. Our results suggest that a two-stage reaction-diffusion system, in which a species involved in the first patterning step modulates a parameter of the second patterning system, could provide an alternative explanation for the initiation of follicle clusters.

For comparison with Fig. 6, Fig. 7 shows a greyscale plot of the steady state activator concentration in a simulation in which $v$ (the decay rate of the inhibitor) was varied sinusoidally with the same wavelength of variation as in Fig. 6(b). We see here that the width of the activator peaks is roughly constant throughout the domain, although the spacing is nonuniform. As in Fig. 6 , the peaks are located on a diagonal array.

\section{Complex pattern formation on patchy domains}

The sinusoidally varying parameters studied in the previous sections would be relevant in a system in which, for example, the activator was degraded by another chemical whose concentration was already spatially varying due to a previous patterning process. Another potential source of inhomogeneities in the reaction-diffusion system is that due to the pattern of differentiation of cells already present in the embryonic field. The states of differentiation of a cell are often essentially discrete and cells of the same type often reside together with sharp boundaries between different cell types (due to differential cell adhesion-see, for example [14]). Thus, we explore now the case where the heterogeneities due to different cell types take the form of distinct patches within the domain. We assume that the boundaries between these patches are sharp, resulting in parameter discontinuities across the boundaries.

As discussed in [26], a discontinuity in a parameter can lead to patterns when the parameters are nowhere in the Turing space. The patterns formed in this case are spatially restricted to the region around the discontinuity. Here, we show that the geometry of the patches can exert an influence on the form of the pattern and can lead, for example, to the production of stripes in a system where spots are normally produced. This suggests that the differences in fish skin patterns between species, for example, might not involve different chemical kinetics, but could arise through different parameter variation on the skin of the different species. We also show that a mixture of stripes and spots can be formed (as on the skin of the 13-lined squirrel, for example, see Fig. 5(d)) and that other patterns such as target patterns can be produced (as in the juvenile Pomacanthus imperator, see Fig. 10(a)).

Fig. 8, shows the late time ${ }^{3}$ activator profile in simulations of the Gierer-Meinhardt model (1) with parameters as given in the legend on the domain $[0,2] \times[0,2]$, with zero flux boundary conditions. In (a), $\mu$ is constant and the parameters are within the Turing space, in (b), $\mu$ takes a stepfunction form with the same value as (a) in the upper half of the domain. The value in the lower half of the domain is outside the Turing space. If $\mu=2.0$ across the whole domain, then the parameters are outside the Turing space and the activator concentration converges everywhere to its homogeneous steady state value (the critical value of $\mu$ is 2.04). We see from the figure that when $\mu=2.5$ everywhere, a pattern of spots is produced. The presence of a patch of $\mu=2.0$, with a straight boundary, however, causes stripes to be produced instead in the region where $\mu=2.5$. The stripes also form in the region where $\mu=2.0$, although their amplitude decays away from the boundary.

The same phenomenon can occur when both patches have parameters within the Turing space (in this case the amplitudes do not decay away from the boundary). Fig. 9(a) shows an example of this in which the boundary between the patches is along a diagonal of the square. This shows that the stripes align with the patch boundary rather than the sides of the square. When the parameter set in one patch is further from bifurcation (further within the Turing space), it initiates spots despite the existence of the discontinuity at the boundary. Thus, the pattern can vary from stripes to spots across the domain, see Fig. 9(b). A local region inside the do-

\footnotetext{
${ }^{3}$ Convergence to steady state is very slow and the time taken for simulation is large, so we wait until the $L_{2}$ norm of the change in $u$ in one time-step, $t=0.01$, is less than the tolerance, which we set to be 0.001 . This happens at $t=192.61$.
} 

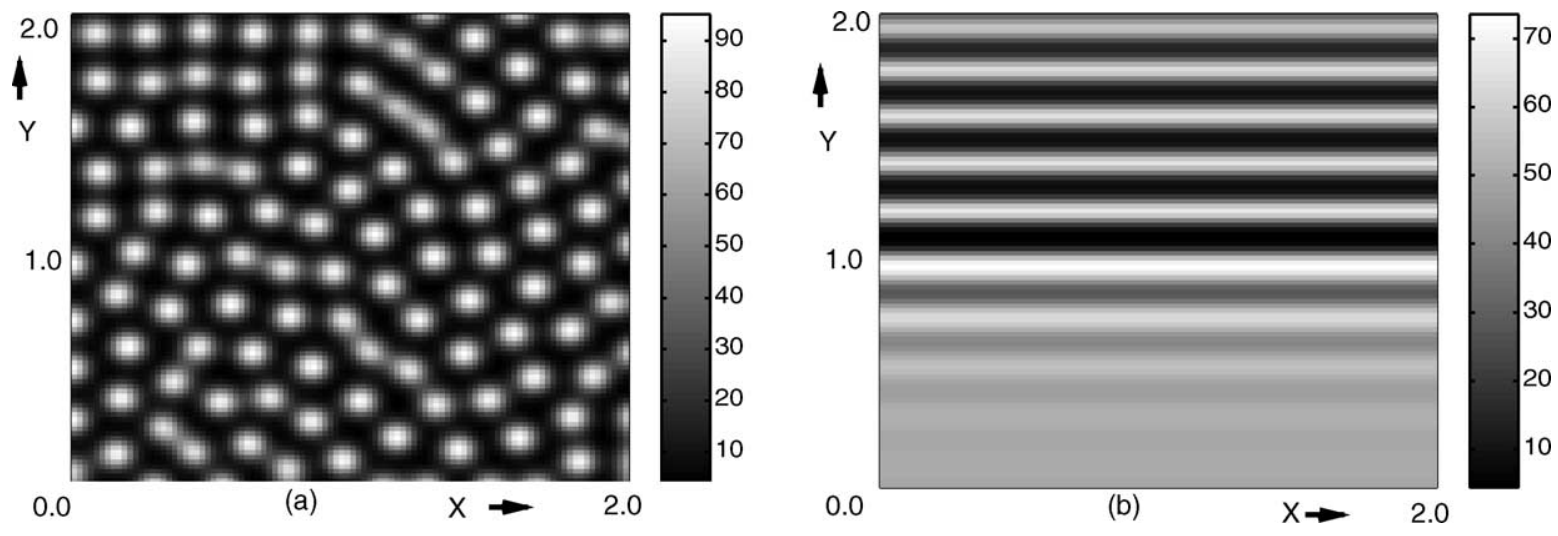

Fig. 8. Plot of the almost steady state activator profiles in simulations of the Gierer-Meinhardt model equations on the domain [0, 2] $\times[0,2]$, with parameters $D_{v}=0.27, v=100.0, r=0.001$ and $d=0.0035$ and zero flux boundary conditions: (a) $\mu=2.5$; (b) $\mu=2.5$ for $y>1.0, \mu=2.0$ for $y<1.0$ [numerical solver-ADI method, timestep $=0.01$, spatial discretization step $=0.02$ ].

main in which a parameter value is different from that in the rest of the domain can lead to a target pattern of rings, similar to that on the skin of the marine fish $P$. imperator, see Fig. 10(a). The local difference could arise naturally due to the local expression of a gene. We show an example of such a pattern in Fig. 9(c), in which $\mu$ takes the value 2.5 on most of the domain, but equals 2.0 on a small square patch in the centre. If the region is at the edge of the domain (for example next to the tail of a fish) then it could lead to a pattern of nested semicircles, as seen on Pomacanthus semicirculatus, see Fig. 10(b). The form of these patterns results from the fact that pattern formation propagates from the boundary of the parameter discontinuity (see [26]). Finally, Fig. 9(d) shows a spatially restricted pattern produced when the parameters are within the Turing space (by which we mean the Turing space for a large zero flux domain) only on a patch in the centre of the domain.

Even if the patterns, such as those in Fig. 9, converge very slowly to a steady state, if the concentration of activator surpasses some threshold for a sustained period in a spatial region, this may be sufficient to trigger differentiation. In general, the changes in pattern which occur at late times tend to take the form of small movements of a few pattern elements or the appearance or disappearance of a pattern element. If we consider that the cells differentiate after sufficient exposure to super-threshold activator, then these rearrangements are likely to have only a minor effect on the pattern.

\section{Discussion}

The patterning of an embryo is naturally hierarchical. Consequently, most patterning processes take place in an environment that is already spatially heterogeneous. At a very early stage the embryo already consists of patches of different cell types and biologically active chemicals are distributed nonuniformly. Turing originally proposed reaction-diffusion as a mechanism for spontaneously generating patterns from random fluctuations about a homogeneous background [30]. Much subsequent work on reaction-diffusion patterning has concentrated on systems with constant coefficients, both because these systems are more mathematically tractable (but still very challenging to solve) and because of the elegant appeal of the symmetry-breaking process.

Much work has focused on pattern selection in nonlinear systems and reaction-diffusion has often been criticised as a model for biological pattern formation because the selection can be very sensitive to perturbations. As illustrated by the results presented here, pattern selection can be greatly influenced by spatial variations in the parameters and so, in modelling biological systems, it may be more relevant to study this more complex case. Attempts to demonstrate Turing patterns in real chemical systems have led to some work on reaction-diffusion systems with graded parameters, since the (quasi-two-dimensional gel) reactors tend to be fed with different chemicals from two opposite boundaries (see, for example [4]). Much of 


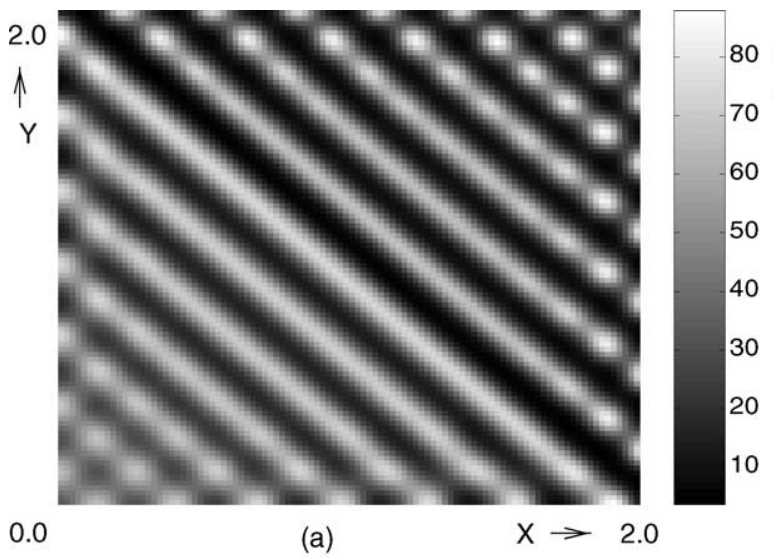

(a)

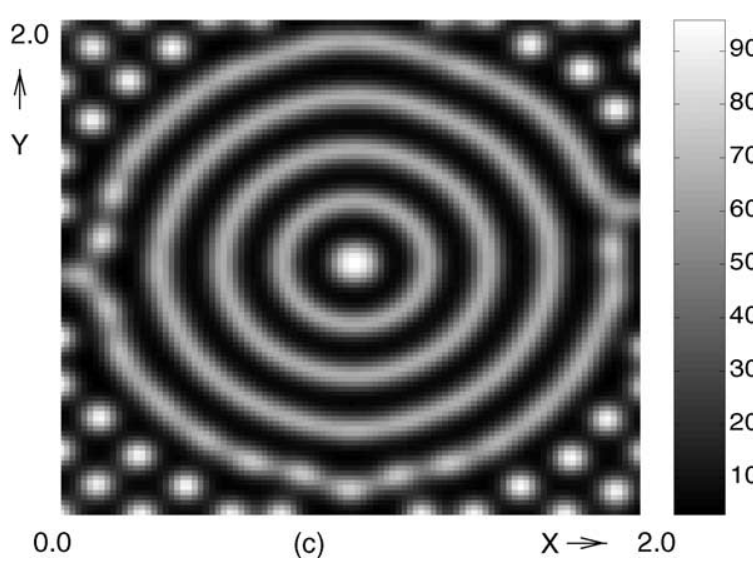

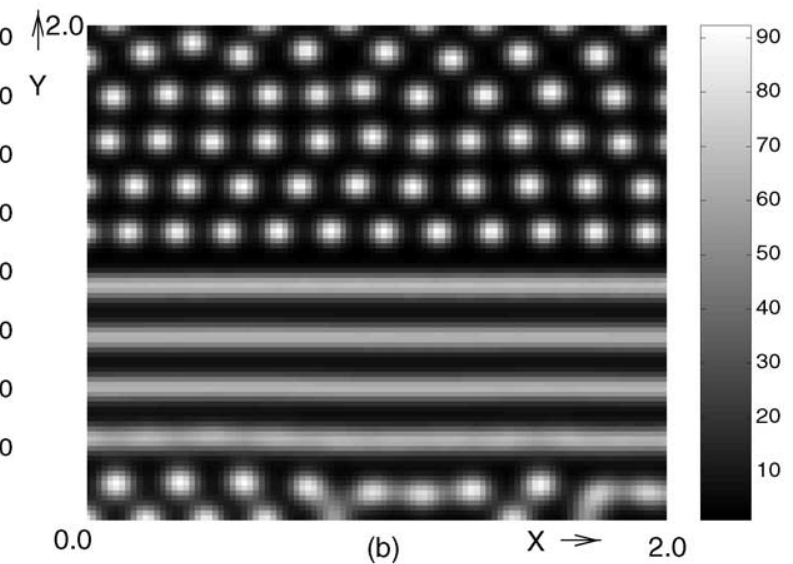

(b)

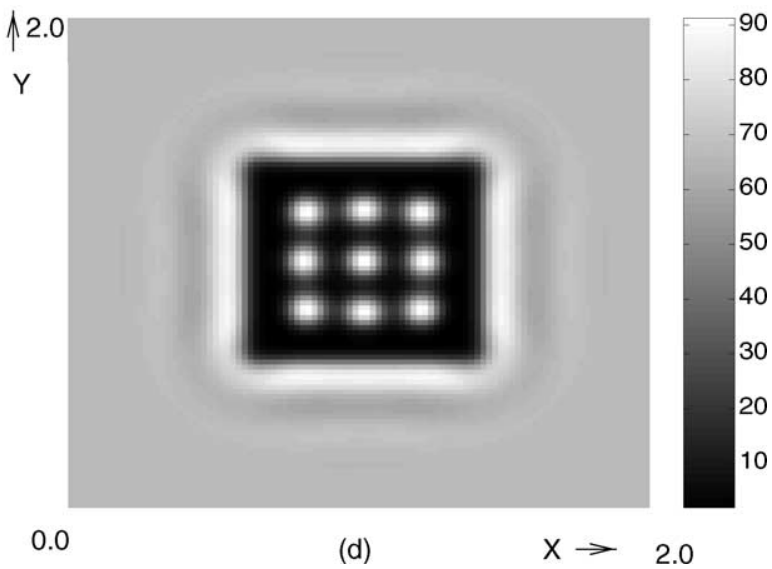

Fig. 9. Plots of the activator profiles in simulations of the Gierer-Meinhardt model equations on the domain $[0,2] \times[0,2]$, with parameters $D_{v}=0.27, v=100.0, r=0.001$ and $d=0.0035$ and zero flux boundary conditions: (a) $\mu=2.4$ for $x+y>2.0$ and $\mu=2.1$ for $x+y \leq 2.0$; (b) $\mu=3.0$ for $y>1.0$ and $\mu=2.5$ for $y \leq 1.0, t=300$; (c) $\mu=2.0$ for $0.9<x<1.1$ and $0.9<y<1.1$ and $\mu=2.5$ elsewhere, $t=105.50$; (d) $\mu=2.5$ for $0.6<x<1.4$ and $0.6<y<1.4$ and $\mu=1.5$ elsewhere [numerical solver-ADI method, timestep=0.01, spatial discretization step $=0.02]$.

this theoretical work relates to the bifurcation structures of the reaction-diffusion systems. More recently, however, periodic spatial forcing has been investigated in a chemical reactor system and it has been shown [11] that this can lead to more regular Turing patterns with fewer defects in two dimensions. In this context, the more complex patterning capabilities of two coupled reaction-diffusion systems have also been investigated [32].

Compared to the experimental chemical reactors, the cellular environment of the embryo can be far more complex and heterogeneous. Cells are nonuniform in their type and density and can produce, sequester and degrade biochemicals in a selective manner. Furthermore, the extracellular matrix may also be heterogeneous and contains factors which modulate the behaviours of putative morphogens [29]. As discussed above, chemicals cannot only modulate the reactions of other chemicals but can also alter their mobility and stability.

In [26], we have shown that spatial variation of parameters in the Gierer-Meinhardt model can lead to the production of patterns when the parameters are everywhere outside the Turing space and that the patterns produced can be spatially restricted. In this paper, we have explored in more detail the ways in 

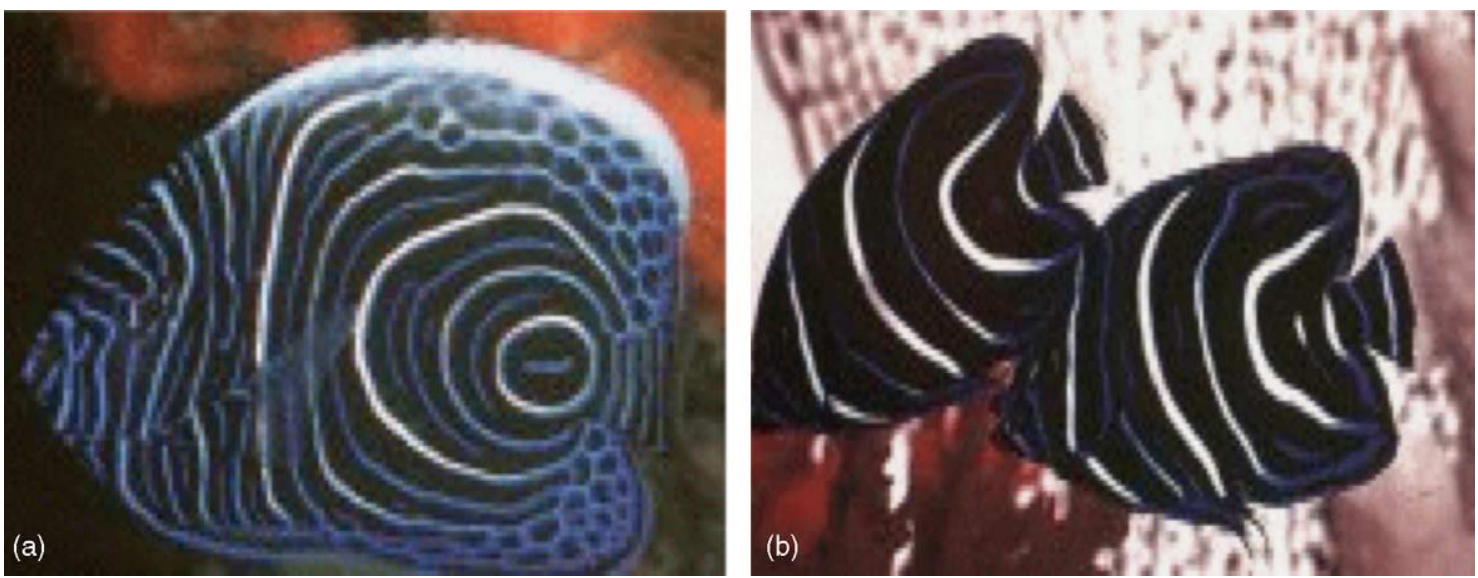

Fig. 10. Pictures of the nested ring-like patterns on skin of the marine angelfish. (a) P. imperator; (b) P. semicirculatus.

which spatially inhomogeneous parameters contribute to the generation of complex patterns. We have looked at the ways in which the various parameters of the Gierer-Meinhardt model system control different aspects of the pattern. The Gierer-Meinhardt model is phenomenological, and so it is not clear that all of the parameters would correspond to simple biophysical quantities in a particular biological application. However, since the diffusion coefficients and decay rates that we have varied are generic parameters it is likely that their effects on the pattern would be qualitatively similar for other model systems. Future work could repeat the analysis of this paper for more realistic models and consider the generality of the results. We conjecture that many features of the work would simply generalise.

Working on a one-dimensional domain, we demonstrated that a pre-pattern in a parameter can lead to a complex pattern of peaks of variable sizes. For certain ratios of pre-pattern wavelength to natural wavelength of the system with constant parameters, we found that the pre-pattern wavelength "fixed" the wavelength of the final pattern, in the sense that only harmonics of the pre-pattern were found in the final pattern (and the pattern was different to that obtained with constant parameters). The pattern formed was independent of the noise in the initial conditions, implying that a pre-pattern in a parameter can enhance the robustness of pattern selection. Thus instead of leading to even more complex possibilities, hierarchical patterning can, in some cases, be more reliable.

We also found that a spatially varying parameter can result in the generation of peaks that move as a wave across the domain. Movement of patterns due to the modulation of a control parameter has been observed before in other patterning systems, for example in Rayleigh-Benard convection, see for example $[15,33]$. Peaks that move as waves have also been seen before in Turing systems where the parameter which is varying in space crosses the Turing bifurcation [17,31]. Here, however, we show that travelling waves of pattern can also occur in a system with a spatially varying parameter, with values that are always within the Turing space. Indeed the patterns concerned have steep "spiky" profiles, characteristic of a system which is far from bifurcation in the highly nonlinear regime. Such behaviour could have important implications for applications in biology. On the one hand, spatio-temporal patterns of gene expression resembling those seen in our simulations have been observed (during the formation of somites, for example see [19]). On the other hand, if small spatial variations in a parameter can cause the peaks of a reaction-diffusion pattern to move, this could cast doubt on the reliability of reaction-diffusion systems as mechanisms for the production of stable spatial patterns. Given that the behaviour of the system will depend on many details such as parameter values and the form of spatial variations, this issue would 
require detailed investigation in any particular application.

In two dimensions, we found that a sinusoidal prepattern in a parameter could give rise to a pattern of large and small spots, like that on the head of the lizard C. fedtschenkoi. These spots can be arranged as a large spot surrounded by several smaller ones, similar to the arrangement of mammalian hair follicles. A hierarchical reaction-diffusion system can thus provide an alternative model of follicle initiation to that proposed by Nagorcka $[23,24]$ and detailed above. We postulate that if the kinetics are such that the system with constant parameters produces a striped pattern, then a sinusoidally varying parameter could lead to a pattern of thick and thin stripes, as seen on the skins of various species. (For a chemotactic model which can give rise to thick and thin stripes see [27].)

The geometry of the parameter patches in two dimensions can have a significant impact on the final pattern formed. If the system with constant parameters gives rise to a pattern of spots, then a straight patch boundary can, nevertheless, trigger a pattern of stripes or a mixed pattern of stripes and spots (for a discussion of the problem of obtaining striped patterns see [20]). A small parameter patch can lead to a target pattern or pattern of nested semicircles, as seen on the skins of fish of the Pomacanthus family.

In summary, we have shown in this paper that spatially varying parameters in reaction-diffusion systems can greatly enhance the range of patterns available to a system (many of which resemble complex patterns seen in biology) whilst at the same time enhancing the robustness of pattern selection.

\section{Acknowledgements}

KMP acknowledges the Joint Research Councils (EPSRC, BBSRC and MRC) for a Lectureship in Bioinformatics. NAMM acknowledges the support of BBSRC (grant no. 50/MMI09729), the Isaac Newton Institute for Mathematical Sciences, University of Cambridge, for an EPSRC-funded Visiting Senior Research Fellowship, and the University of Sheffield for the Graves Research Fellowship in Medical Research. PKM acknowledges support from a Royal Society Leverhulme Trust Senior Research Fellowship and thanks the National Centre for Theoretical Sciences,
Taiwan and Hokkaido University, Sapporo, for partial support.

\section{Appendix A. Matched asymptotic analysis of the Gierer-Meinhardt system}

To determine analytically the effect on patterning of each parameter in the Gierer-Meinhardt model (Eq. (1)) we assume that $D_{u}$ is very much smaller than $D_{v}$ and set $D_{u}=\epsilon^{2} \times D_{v}$, where $\epsilon$ is a small parameter. In one spatial dimension, steady state solutions of Eq. (1) satisfy the following equations and boundary conditions:

$r\left(1+\frac{u^{2}}{v}\right)-\mu u+\epsilon^{2} D_{v} u_{x x}=0$,

$r u^{2}-v v+D_{v} v_{x x}=0$ for $x \in(0,1)$,

$u_{x}=0 \quad$ for $x=0,1, \quad v_{x}=0 \quad$ for $x=0,1$.

In order to obtain a solution of these equations that is $\mathrm{O}(1)$ on most of the domain, we must make the further assumption that $r$ is small. This is often the case for the (biologically plausible) parameters that are used in numerical simulations, since large values of $r$ tend to put the parameters out of the Turing space. We initially assume that $r$ is $\mathrm{O}(\epsilon)$ and set $r=\epsilon \alpha$, where $\alpha$ is $\mathrm{O}(1)$; we later look for a solution when $r$ is $\mathrm{O}(1)$.

We seek a solution of Eq. (A.1) for $u$ and $v$ in terms of a series expansion in $\epsilon$. However it is not possible to construct a regular series expansion which satisfies the differential equations and both boundary conditions. We thus consider an "outer expansion" which is regular and satisfies the equations in most of the domain together with "inner expansions" which match with the outer solution and satisfy the equations in small boundary layer regions of the domain. In these regions the coefficients of the series solution are given in terms of a rescaled spatial coordinate.

Considering first the outer expansion, we have

$u=u_{0}(x)+\epsilon u_{1}(x)+\epsilon^{2} u_{2}(x)+\cdots$,

$v=v_{0}(x)+\epsilon v_{1}(x)+\epsilon^{2} v_{2}(x)+\cdots$,

where $u_{0}(x), u_{1}(x), \ldots$ and $v_{0}(x), v_{1}(x), \ldots$ are twice differentiable functions of $x$. Substituting into (A.1) and 
collecting terms of $\mathrm{O}(1)$, we find

$u_{0}=0, \quad D_{v}\left(v_{0}\right)_{x x}-v v_{0}=0$.

The latter equation implies

$v_{0}=A \cosh \left(\frac{x-x_{0}}{\lambda_{v}}\right)$,

where $A$ and $x_{0}$ are constants. From consideration of terms of order $\epsilon$ we obtain ${ }^{4}$

$u_{1}=\frac{\alpha}{\mu}, \quad v_{1}=0$.

We now consider the inner solution at an arbitrary point $x_{1}$ in the domain. We re-scale the spatial coordinate and consider a solution $u=\tilde{u}(X)$ and $v=\tilde{v}(X)$, where $X=\left(x-x_{1}\right) / \epsilon^{\beta}$ for some positive exponent $\beta$. We find that $\beta=1$ yields a solution of the form

$\tilde{u}(X)=\frac{1}{\epsilon}\left(\bar{u}_{0}(X)+\epsilon \bar{u}_{1}(X)+\cdots\right)$,

$\tilde{v}(X)=\bar{v}_{0}(X)+\epsilon \bar{v}_{1}(X)+\cdots$.

Substituting this into (A.1) and looking at the leading order terms yields

$\alpha \frac{\bar{u}_{0}(X)^{2}}{\bar{v}_{0}(X)}-\mu \bar{u}_{0}(X)+D_{v}\left(\bar{u}_{0}\right)_{X X}=0, \quad\left(\bar{v}_{0}\right)_{X X}=0$.

In order to match with the outer solution, $\lim _{X \rightarrow \pm \infty} \bar{v}_{0}(X)$ must be finite. Together with the second equation of (A.7) this implies

$\bar{v}_{0}(X)=v_{*}$,

where $v_{*}$ is a constant.

Substituting into the first equation of (A.7), this gives

$\alpha \frac{\bar{u}_{0}^{2}}{v_{*}}-\mu \bar{u}_{0}+D_{v}\left(\bar{u}_{0}\right)_{X X}=0$.

Together with the matching conditions, which imply that $\bar{u}_{0}(X)$ tends to 0 as $X \rightarrow \pm \infty$, this has solution

$\bar{u}_{0}(X)=3 \frac{\mu v_{*}}{2 \alpha} \operatorname{sech}^{2}\left(\sqrt{\frac{\mu}{D_{v}}} \frac{X}{2}\right)$.

\footnotetext{
${ }^{4}$ While $v_{1}$ satisfies an equation similar to that for $v_{0}$, it can be shown that the solution of this equation is actually $v_{1}=0$.
}

Substituting the inner expansion into (A.1) and collecting terms of order $1 / \epsilon$ in the inhibitor equation yields

$$
D_{v}\left(\bar{v}_{1}\right)_{X X}=-\alpha \bar{u}_{0}^{2} .
$$

Now the change in $v_{x}$ across the boundary layer is

$$
\begin{aligned}
\Delta v_{x}= & \int_{x_{1}^{-}}^{x_{1}^{+}} v_{x x} \mathrm{~d} x \\
= & \int_{-\infty}^{\infty} \frac{1}{\epsilon^{2}} v_{X X} \epsilon \mathrm{d} X+\text { smaller terms } \\
= & \int_{-\infty}^{\infty}\left(\bar{v}_{1}\right)_{X X} \mathrm{~d} X+\text { smaller terms } \\
& \times \int_{-\infty}^{\infty}-\frac{\alpha}{D_{v}} \bar{u}_{0}^{2} \mathrm{~d} X+\text { smaller terms }
\end{aligned}
$$

Substituting in our expression for $\bar{u}_{0}$ from (A.10) and integrating gives

$\Delta v_{x}=-\frac{6 \mu^{3 / 2} v_{*}^{2}}{\alpha D_{v}^{1 / 2}}$.

We can thus completely determine the leading order outer solution for the inhibitor if we know the location of the boundary layers and if we know the value of $v_{*}$ at the leftmost peak (this and the left hand boundary condition determine the solution to the left of the first boundary layer and we use this to match the solution and its derivative with the solution to the right of the boundary layer). The right hand boundary condition will then determine $v_{*}$ if we know the positions of the boundary layers. Thus the location of the boundary layers is sufficient to fully determine the solution. Alternatively, we can use an integral condition (obtained from the differential equation for $v$ ) to determine $v_{*}$. We illustrate this for an example solution with $n$ boundary layers at $x=1 / 2 n, 3 / 2 n, \ldots,(2 n-1) / 2 n$.

Integrating the second equation of (1) and using the zero flux boundary conditions, we obtain

$$
\int_{0}^{1}\left(r u^{2}-v v\right) \mathrm{d} x=0 .
$$


Now, with a boundary layer at $x=1 / 2 n$, the outer solution for $v$ has a leading order term given by

$v= \begin{cases}A \cosh \left(\frac{x}{\lambda_{v}}\right), & x \in\left[0, \frac{1}{2 n}\right] \\ B \cosh \left(\frac{1 / n-x}{\lambda_{v}}\right), & x \in\left[\frac{1}{2 n}, \frac{1}{n}\right],\end{cases}$

where $A$ and $B$ are constants satisfying $v_{*}=$ $A \cosh \left(1 / 2 n \lambda_{v}\right)=B \cosh \left(1 / 2 n \lambda_{v}\right)$. Thus, to leading order,

$v \int_{0}^{1} v \mathrm{~d} x=2 n v_{*} \sqrt{D_{v} v} \tanh \left(\frac{1}{2 n \lambda_{v}}\right)$.

Now the leading order term in the outer expansion for $u$ is $\mathrm{O}(\epsilon)$ and hence the contribution to $\int_{0}^{1} r u^{2} \mathrm{~d} x$ will be $\mathrm{O}\left(\epsilon^{3}\right)$, whereas the inner expansion for $u$ is $\mathrm{O}(1 / \epsilon)$, the width of the boundary layer is at least $\mathrm{O}(\epsilon)$ and hence the contribution from the inner expansion will be at least $\mathrm{O}(1)$. Thus, using the first equation of (A.6) and Eq. (A.10), we obtain, to leading order,
Thus, substituting (A.16) and (A.17) into (A.14), we obtain

$v_{*}=\frac{r \xi}{3 \mu} \tanh \left(\frac{1}{2 n \lambda_{v}}\right)$.

We wish to compare our matched asymptotic solutions to the numerical solution. Our analysis does not allow us to determine the location of the boundary layers. If, however, we know the location and height of the leftmost inhibitor peak, we can calculate its form and the height and the form of the activator peak. Fig. A.1 shows the results of simulation of the Gierer-Meinhardt model equations with parameters $D_{v}=0.027, v=1.1, \mu=1.0, \epsilon=0.01$ and $r=0.01$ (i.e. $\alpha=1.0$ ) on the domain $[0,1]$ with zero flux boundary conditions. The initial values of $u$ and $v$ are random perturbations about the homogeneous steady state values. We find that the leftmost inhibitor peak is at $x \approx 0.327$ and has height $v_{*} \approx 0.3295$. We use these values in the leading order analytical outer solution for the inhibitor concentration (using the inner solution to give the condition on the change in the derivative across the boundary layer and to show that the change in the inhibitor concentration is zero to leading order).

$$
\int_{0}^{1} r u^{2} \mathrm{~d} x=n \int_{-\infty}^{\infty} \frac{9 \mu^{2} v_{*}^{2}}{4 r} \operatorname{sech}^{4}\left(\sqrt{\frac{\mu}{D_{v}}} \frac{X}{2}\right) \epsilon \mathrm{d} X=\frac{9 n \mu^{2} v_{*}^{2}}{2 \alpha} \sqrt{\frac{D_{v}}{\mu}} \int_{-\infty}^{\infty} \operatorname{sech}^{4} y \mathrm{~d} y=\frac{9 n \mu^{2} v_{*}^{2}}{2 \alpha} \sqrt{\frac{D_{v}}{\mu}} \frac{4}{3} .
$$
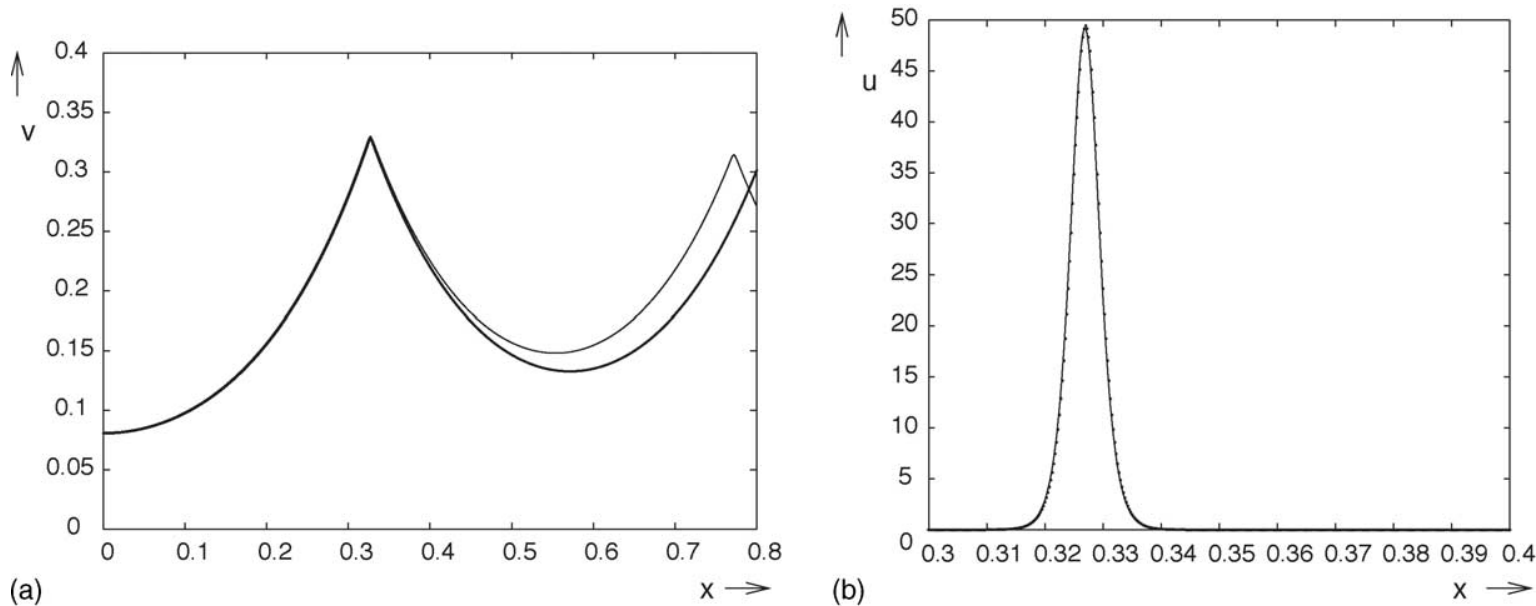

Fig. A.1. (a) Plot of the analytical (thick line) estimate of the leading order outer solution to the inhibitor concentration compared with the numerical solution (thin line). (b) Plot of the analytical boundary layer solution (dotted line) of the activator equation compared with the numerical solution (solid line) [numerical solver - Nag library routine: D03PCF, number of discretization points $=1001$, tolerance $=10 \mathrm{E}-07$, initial conditions $\pm 10 \%$ noise around the uniform steady state]. 
The analytical solution shown in Fig. A.1(a) to the right of the (first) boundary layer is given by $0.3295 \cosh \left((x-0.327) / \lambda_{v}\right)-0.3016 \sinh ((x-$ $\left.0.327) / \lambda_{v}\right)$. When we match the solution instead with its numerical value at its turning point, we obtain the solution $0.3306 \cosh \left((x-0.327) / \lambda_{v}\right)-$ $0.2956 \sinh \left((x-0.327) / \lambda_{v}\right)$. In Fig. A.1(a), we show the resulting analytical solution on the domain to the left of the second boundary layer (thick line) compared with the numerical solution (thin line). The solution becomes inaccurate towards the second boundary layer. This is due to a small error in the values used for the matching conditions. Thus we see that a small discrepancy in the coefficients of the cosh and sinh parts of the solution (caused by a small error in the matching values of $v_{*}$ and $\Delta v_{x}$ ) is enough to lead to a significant discrepancy in the solution far from the boundary layer.

To leading order, the activator concentration is zero outside the boundary layer and has a very high peak in the boundary layer. In Fig. A.1(b), we show that there is excellent agreement between the analytical form of this solution (assuming the value of $v_{*}$ obtained from the numerical inhibitor solution) and the numerical solution (solid line) on a small region of the domain that includes the left-hand boundary layer.

Our analysis shows that when $d \equiv D_{u} / D_{v}$ is $\mathrm{O}\left(\epsilon^{2}\right)$, the width of the activator peaks is proportional to the diffusion wavelength of the activator $\lambda_{u} \equiv \sqrt{D_{u} / \mu}$. The length-scale of the inhibitor pattern (which gives us an idea of the spacing of the activator peaks) is of the order of the diffusion wavelength of the inhibitor $\lambda_{v} \equiv \sqrt{D_{v} / v}$. If we assume that the boundary layers occur at locations $x_{i}$, with spacings $x=x_{i+1}-x_{i}$ satisfying $x \sim \lambda_{v}$, then on each side of each boundary layer we have

$v_{*} \sim A$,

$\left|v_{x}\right| \sim \frac{A}{\lambda_{v}}$,

and hence $\Delta v_{x} \sim v_{*} / \lambda_{v}$ (since the sign of the gradient of $v$ changes in the boundary layer, we know that the leading order terms in $v_{x}$ on either side of the boundary layer will contribute to $\Delta v_{x}$ ). Substituting the expression for $\Delta v_{x}$ from (A.11), we obtain

$\frac{v^{1 / 2}}{D_{v}^{1 / 2}} v_{*} \sim \frac{\mu^{3 / 2} v_{*}^{2} d^{1 / 2}}{r D_{v}^{1 / 2}}$, and hence

$v_{*} \sim A \propto \frac{r \xi}{\mu}$.

(This also agrees with the exact solution that we obtain for a single boundary layer at $x=1 / 2$, provided that $1 / \lambda_{v}$ is $O(1)$, which is necessary for the single boundary layer solution to be likely to arise in practice.) From (A.6) and (A.10) we see that this implies that the heights of the activator peaks are proportional to $\xi=\sqrt{D_{v} v / D_{u} \mu}$.

We look now for a perturbation solution when $r$ is $\mathrm{O}(1)$. We find that the Gierer-Meinhardt model equations without the constant term $+r$ in the activator equation (see Eq. (1)), exhibit a symmetry under the transformation $r \rightarrow k r, v \rightarrow k v, u \rightarrow u$. Hence we expect $v \propto r$. We also find in simulations and in the above perturbation analysis for small $r$ that $v$ is roughly proportional to $r$ in the full Gierer-Meinhardt model system with the added constant term. This leads us to look for an outer expansion in which $v$ is $\mathrm{O}(1 / \epsilon)$ when $r$ is $\mathrm{O}(1)$, since $v$ was $\mathrm{O}(1)$ when $r$ was $\mathrm{O}(\epsilon)$. The analysis follows as before and we find an outer solution

$u=u_{0}+\epsilon u_{1}+\cdots, \quad v=\frac{1}{\epsilon}\left(v_{0}+\epsilon v_{1}+\cdots\right)$,

where

$u_{0}=\frac{r}{\mu}, \quad v_{0}=A \cosh \left(\frac{x-x_{0}}{\lambda_{v}}\right)$,
$u_{1}=\frac{r^{3}}{\mu^{2} A} \operatorname{sech}\left(\frac{x-x_{0}}{\lambda_{v}}\right), \quad v_{1}=\frac{r^{3}}{\mu^{2} v}$,

and $A$ and $x_{0}$ are constants. The inner solution at a boundary layer point $x_{1}$ is given by

$v=v_{*}+\cdots, \quad u=\frac{3 \mu v_{*}}{2 r} \operatorname{sech}^{2}\left(\frac{x-x_{1}}{2 \lambda_{u}}\right)+\cdots$,

where $v_{*}$ is a constant of $\mathrm{O}(1 / \epsilon)$. To satisfy matching conditions with the outer solutions on either side of the boundary layer,

$v_{*}=\frac{A}{\epsilon} \cosh \left(\frac{x_{1}-x_{0}}{\lambda_{v}}\right)$. 
We also obtain the same condition as before on the discontinuity in the derivative of $v$,

$\Delta v_{x}=-\frac{6 \mu^{3 / 2} \epsilon v_{*}^{2}}{r D_{v}^{1 / 2}}$.

The leading order forms (both inner and outer) of these solutions are exactly the same as for $r=\mathrm{O}(\epsilon)$ up to the location of the boundary layers, provided that we can still assume that the boundary layers occur at separations satisfying $x \sim \lambda_{v}$. Thus the conclusions that we came to above about the dependence of the widths, heights and separation of the parameters should still hold for $r=\mathrm{O}(1)$.

\section{Appendix B}

We consider the case $d=\mathrm{O}\left(\epsilon^{2}\right), r=\mathrm{O}(1)$, but the analysis follows similarly for $r=\mathrm{O}(\epsilon)$. We look at the inner expansion in a boundary layer around the point $x=x_{0}: u=(1 / \epsilon) \bar{u}\left(\left(x-x_{0}\right) / \epsilon\right), v=$ $(1 / \epsilon) \bar{v}\left(\left(x-x_{0}\right) / \epsilon\right)$. To leading order,

$0=D_{v}\left(\bar{v}_{0}\right)_{X X}$,

$\left(\bar{u}_{0}\right)_{t}=r \frac{\bar{u}_{0}^{2}}{\bar{v}_{0}}-\mu\left(\bar{u}_{0}\right)+D_{v}\left(\bar{u}_{0}\right)_{X X}$.

Together with the boundary (matching) conditions for $v$, the first equation implies that $v=v_{*}$, where $v_{*}$ is a constant of $\mathrm{O}(1 / \epsilon)$. Thus

$\left(\bar{u}_{0}\right)_{t}=r \frac{\bar{u}_{0}^{2}}{\epsilon v_{*}}-\mu\left(\bar{u}_{0}\right)+D_{v}\left(\bar{u}_{0}\right)_{X X}$.

Now, if we let $w=1-r \bar{u}_{0} / \mu \epsilon v_{*}, t^{\prime}=\mu t$ and $X^{\prime}=$ $\sqrt{\mu / D_{v}} X$, then

$w_{t^{\prime}}=w(1-w)+w_{X^{\prime} X^{\prime}}$,

which is Fisher's equation.

\section{References}

[1] R.A. Barrio, C. Varea, J.L. Aragon, P.K. Maini, A twodimensional numerical study of spatial pattern formation in interacting Turing systems, Bull. Math. Biol. 61 (1999) 83505.

[2] F. Brümmer, G. Zempel, P. Buehle, J.-C. Stein, D.F. Huelser, Retinoic acid modulates gap junctional permeability: a com- parative study of dye spreading and ionic coupling in cultured cells, Exp. Cell Res. 196 (1991) 158-163.

[3] K.M. Cadigan, Regulating morphogen gradients in the drosophila wing, Sem. Cell Dev. Biol. 13 (2002) 83-90.

[4] V. Castets, E. Dulos, J. Boissonade, P. De Kepper, Experimental evidence of a sustained standing Turing-type nonequilibrium chemical pattern, Phys. Rev. Lett. 64 (1990) 2953-2956.

[5] C.N.D. Coelho, R.A. Kosher, A gradient of gap junctional communication along the anterior-posterior axis of the developing chick limb bud, Dev. Biol. 48 (1991) 529-535.

[6] J.R. Collier, Spatial and propagating patterns in embryology, DPhil Thesis, University of Oxford, 1997.

[7] E.J. Crampin, E.A. Gaffney, P.K. Maini, Reaction and diffusion on growing domains: scenarios for robust pattern formation, Bull. Math. Biol. 61 (1999) 1093-1120.

[8] M.C. Cross, P.C. Hohenberg, Pattern formation outside of equilibrium, Rev. Mod. Phys. Part 265 (3) (1993).

[9] A. De Wit, Spatial patterns and spatiotemporal dynamics in chemical systems, Adv. Chem. Phys. 109 (1999) 435513.

[10] A. Doelman, T.J. Kaper, H. van der Ploeg, Spatially periodic and aperiodic multi-pulse patterns in the one-dimensional Gierer-Meinhardt equation, Meth. Appl. Anal. 8 (2001) 387414.

[11] M. Dolnick, I. Berenstein, A.M. Zhabotinsky, I.R. Epstein, Spatial periodic forcing of Turing structures, Phys. Rev. Lett. 87 (2001) article no. 238301.

[12] A. Gierer, H. Meinhardt, A theory of biological pattern formation, Kybernetik 12 (1972) 30-39.

[13] S.F. Gilbert, Developmental Biology, 4th ed., Sinauer, 1994.

[14] J.A. Glazier, F. Graner, Simulation of the differential adhesion driven rearrangement of biological cells, Phys. Rev. E47 (1993) 2128-2154.

[15] G. Hartung, F.H. Busse, I. Rehberg, Time-dependent convection induced by broken spatial symmetries, Phys. Rev. Lett. 66 (1991) 2742-2745.

[16] R. Kapral, K. Showalter, Chemical Waves and Patterns, Kluwer, 1995.

[17] L. Kramer, E. Ben-Jacob, H. Brand, M. Cross, Wavelength selection in systems far from equilibrium, Phys. Rev. Lett. 49 (1982) 1891-1894.

[18] P.K. Maini, K.J. Painter, H.N.P. Chau, Spatial pattern formation in chemical and biological systems, J. Chem. Soc., Faraday Trans. 93 (1997) 3601-3610.

[19] M.J. McGrew, J.K. Dale, S. Fraboulet, O. Pourquie, The lunatic Fringe gene is a target of the molecular clock linked to somite segmentation in avian embryos, Curr. Biol. 8 (1998) 979-982.

[20] H. Meinhardt, Growth and patterning - dynamics of stripe formation, Nature 376 (1995) 722-723.

[21] J.D. Murray, Parameter space for Turing instability in reactiondiffusion mechanisms: a comparison of models, J. Theor. Biol. 98 (1982) 143-163.

[22] J.D. Murray, Mathematical Biology I: An Introduction, 3rd ed., Springer-Verlag, 2002.

[23] B.N. Nagorcka, The reaction-diffusion (RD) theory of wool (hair) follicle initiation and development. I. Primary follicles, Aust. J. Agric. Res. 46 (1995) 333-355. 
[24] B.N. Nagorcka, The reaction-diffusion (RD) theory of wool (hair) follicle initiation and development. II. Original secondary follicles, Aust. J. Agric. Res. 46 (1995) 357-378.

[25] C. Neumann, S. Cohen, Morphogens and pattern formation, Bioessays 19 (1997) 721-729.

[26] K.M. Page, P.K. Maini, N.A.M. Monk, Pattern formation in spatially heterogeneous Turing reaction-diffusion models, Physica D 181 (2002) 80-101.

[27] K.J. Painter, P.K. Maini, H.G. Othmer, Stripe formation in juvenile Pomacanthus explained by a generalized Turing mechanism with chemotaxis, PNAS 96 (1999) 5549-5554.

[28] D. Peaceman, J.H.H. Rachford, The numerical solution of parabolic and elliptic differential equations, J. Soc. Ind. Appl. Math. 3 (1955) 28-41.
[29] J. Taipale, J. Keski-Oja, Growth factors in the extracellular matrix, FASEB J. 11 (1997) 51-59.

[30] A.M. Turing, The chemical basis of morphogenesis, Phil. Trans. R. Soc. 237 (1952) 37-72.

[31] J.-P. Voroney, A.T. Lawniczak, R. Kapral, Turing pattern formation in heterogeneous media, Physica D 99 (1996) 303317.

[32] L. Yang, M. Dolnick, A.M. Zhabotinsky, I.R. Epstein, Spatial resonances and superposition patterns in a reaction-diffusion model with interacting Turing modes, Phys. Rev. Lett. 88 (2002) article no. 208303.

[33] W. Zimmermann, R. Schmitz, Hopf bifurcation by frustrated drifts, Phys. Rev. E 53 (1996) R1321-R1325. 